\title{
TRAF1/C5 rs3761847 SNP Is Associated with Severe Pattern of Rheumatoid Arthritis in Greek Patients
}

\author{
Alexandros Sarantopoulos ${ }^{1 *}$, Ioannis Theodorou ${ }^{2}$, Panagiota Boura ${ }^{1}$ \\ ${ }^{1}$ Clinical Immunology Unit, $2^{\text {nd }}$ Department of Internal Medicine, Aristotle University of Thessaloniki, Thessaloniki, \\ Greece \\ '2Laboratoire d'Histocompatibilité et Immunogénétique, Département d'Immunologie, Groupe Hospitalier Pitié \\ Salpetrière, Paris, France \\ Email: *alexsar@med.auth.gr
}

Received 8 October 2015; accepted 16 February 2016; published 19 February 2016

Copyright (C) 2016 by authors and Scientific Research Publishing Inc.

This work is licensed under the Creative Commons Attribution International License (CC BY). http://creativecommons.org/licenses/by/4.0/

c) $\underset{\mathrm{EY}}{\mathrm{B}}$ Open Access

\section{Abstract}

The era of whole genome study analysis has introduced a profound research in the genetics of autoimmune diseases. Some of the new genetic loci that have been associated with the development or the severity of autoimmune diseases have been thoroughly studied, conferring a more detailed understanding of disease pathophysiology. Furthermore, single nucleotide polymorphisms (SNPs) have been described not only in coding regions of the human genome but also in non-coding areas (introns), the importance of which has not been yet clarified. Over the last years, such an SNP has been associated with the development of rheumatoid arthritis, the most frequent autoimmune disease. This SNP is at the position 122730060 of chromosome 9 in the TRAF1/C5 region and consists of a substitution of the nucleotide base guanine (G) - which is considered the ancestral phenotype-by alanine (A). It has been indicated that $G$ is the aggravating nucleotide, and that $G / G$ is the disease predisposing phenotype, conferring $>1.3 \times$ risk for RA. On this background, we performed a genome study on a Greek population of northern Greece (Macedonia) in order to identify the association of this SNP with RA in our group.

\section{Keywords}

Rheumatoid Arthritis, Immunogenetics, Disease Pattern

\section{Introduction}

Rheumatoid Arthritis (RA) is the most frequent systemic autoimmune disease, affecting approximately $1 \%$ of ${ }^{*}$ Corresponding author.

How to cite this paper: Sarantopoulos, A., Theodorou, I. and Boura, P. (2016) TRAF1/C5 rs3761847 SNP Is Associated with Severe Pattern of Rheumatoid Arthritis in Greek Patients. Open Journal of Rheumatology and Autoimmune Diseases, 6, 10-12. http://dx.doi.org/10.4236/ojra.2016.61002 
global population. The 2010 EULAR/ACR (European League Against Rheumatism/American College of Rheumatology) diagnostic criteria can set the diagnosis in an early stage of RA, providing access of RA patients to available conventional synthetic or biological Disease Modifying Anti-Rheumatic Drug (DMARD) therapy. TNF $\alpha$ blockade application in clinical practice has imposed an immense investigation on determining biomarkers that could predict better response to treatment in Rheumatoid Arthritis patients. The whole genome scan era has permitted the identification of single nucleotide polymorphisms (SNPs) that predispose to the development of RA but no further studies have been conducted to reveal any possible susceptibility of these genetic loci with pattern of disease. Furthermore, no therapeutic intervention has been proposed by genetic factors associated to any way with RA, indicating that the genetic findings cannot so far be directly associated with RA pathophysiology and therefore treatment. On the other hand, economic burden of anti-TNF $\alpha$ treatment poses persistently the identification of response predictors in order to better apply individualized treatment.

\section{Materials and Methods}

On this regard, we performed a genotype analysis of 172RA patients and 95healthy adult individuals (control group) from Northern Greece provisioned by the Clinical Immunology Unit of the $2^{\text {nd }}$ Department of Internal Medicine of Aristotle University of Thessaloniki, Greece. All subjects had signed an informed consent for their genotyping. The observational nature of our study did not require an ethics committee approval. Genotypic analysis was performed at the Histocompatibility and Immunogenetic Laboratory of the Department of Immunology of INSERM UMRS 945, Pitié-Salpêtrière Hospital, Paris, France. An AB (Applied Biosystems) TaqMan ${ }^{\circledR}$ SNP Genotyping Assay was used for the assessment of the investigated SNP on a AB 7900HT Fast RealTime Polymerase Chain Reaction (RT-PCR) System.

\section{Results}

The primary target of our study was to investigate whether findings indicating a correlation of rs3761847SNP to RA manifestation, conducted in Northern Europe and American population [1], had a similar burden on Greek patients, since it is known that Greek patients differ genetically from northern Europe populations in regard to genomic profile associated to RA [2]. Our initial findings did not identify any difference of genetic profile between RA patients and healthy volunteers (Table 1), indicating that in Greek population, in contrast to wide population studies conducted in different Caucasian groups [3], TRAF1/C5 rs3761847 variant does not predispose to RA manifestation. These results are in accordance to studies that have been conducted in Asian populations [4], with whom Mediterraneans share more common genetic profiles.

Nevertheless, when we sub grouped our patients on those receiving conventional non-biological DMARD therapy $(\mathrm{n}=134)$ and those receiving anti-TNF $\alpha$ treatment $(\mathrm{n}=38)$, we underlined a statistically important difference of $\mathrm{A} / \mathrm{G}$ allele rs3761847 burden between the 2 groups (A/G $61.3 \% / 38.7 \%$ on the non-biological DMARD group vs $50.88 \% / 49.12 \%$ on the anti-TNF $\alpha$ group-Table 2). Since RA patients with long-standing disease receiving non-biologic agents express a less severe RA pattern in respect to those enrolled to anti-TNF $\alpha$ treatment, augmented incidence of allele A in rs3761847 on the first group might imply a protective role of allele A on Greek RA patients. According to our knowledge, our findings have never been observed in other ethnical

Table 1. Frequency of alleles A/G of rs3761847 in RA patients and in controls.

\begin{tabular}{cccc}
\hline & RA Patients $(\mathrm{n}=172)$ & Controls $(\mathrm{n}=95)$ & $p$ value \\
\hline $\mathrm{A}$ & 58.85 & 59.02 & NS \\
$\mathrm{G}$ & 41.15 & 40.98 & NS \\
\hline
\end{tabular}

Table 2. Frequency of alleles A/G of rs3761847 in RA patients receiving synthetic DMARDs or anti-TNF $\alpha$ treatment.

\begin{tabular}{cccc}
\hline & DMARDs $(\mathrm{n}=134)$ & Anti-TNF $\alpha(\mathrm{n}=38)$ & $p$ value \\
\hline $\mathrm{A}$ & 61.3 & 50.88 & $<0.05$ \\
$\mathrm{G}$ & 38.7 & 49.12 & $<0.05$ \\
\hline
\end{tabular}


groups and are of special interest, since although TRAF1/C5 rs3761847 variant in Greek population does not predispose to the development of RA, on RA patients though, it is associated with reduced disease severity, as augmented proportion of allele A was observed in patients receiving conventional DMARD therapy. Further analysis of our cohort of RA patients receiving anti-TNF $\alpha$ agents did not point out any correlation of this SNP to anti-TNF $\alpha$ response parameters (seropositivity, initial disease activity).

\section{Discussion}

This study is the first to identify an attenuating role of allele A of rs3761847SNP on RA pattern. On molecular basis, our findings could presume that the specific SNP in Greek patients is not potent enough to scatter a deviation of the immune response and contribute to the development of RA, but in RA patients, where the immune response is reorganized by other genetic factors in promoting the disease, only there can this SNP interfere in an unknown pattern with a deviated immune response and express a protective role against the development of RA.

The fact that this SNP is situated in a non coding area of the human genome (intron) raises the question whether these silent areas have after all a functional role in human cell homeostasis [5]. This question has been raised by genetics, and is overwhelmed by studies that like ours identify associations of these loci with human disease.

The clinical equivalent of the correlation observed in our study is that patients presenting the specific SNP phenotype are more likely to sustain prolonged remission with conventional synthetic DMARD therapy, sparing them from more expensive regiments, such as biologic therapies. Given the fact that biologic therapies have a high economical cost of commercial price and follow-up of RA patients receiving them, it is evident that both these costs can be avoided by designing a much cheaper synthetic DMARD combination therapy for RA patients based on their genetic profile.

\section{Conclusion}

This study indicates that RA patients with a specific SNP are most likely to undergo sustained remission with a combination of less expensive, synthetic DMARD therapies. In an era of long lasting economical crisis, affecting especially Greece, any genetic indicator of response to therapy is essential in designing cost-benefit patienttailored treatment. Nevertheless, on the era of almost 10 clinical applied biologic DMARDs, targeting totally different pathophysiological RA pathways, whole genome scan analysis may still identify genomic patients' profiles predicting a higher likelihood of response to any of these agents.

\section{Acknowledgements}

We thank Philippe Pellet who monitored the execution of the genomic analysis, and the patients and physicians who participated in this study.

\section{Conflict of Interest}

None declared.

\section{References}

[1] Plenge, R.M., Seielstad, M., Padyukov, L., et al. (2007) TRAF1-C5 as a Risk Locus for Rheumatoid Arthritis-A Genome Wide Study. New England Journal of Medicine, 357, 1199-1209. http://dx.doi.org/10.1056/NEJMoa073491

[2] Ioannidis, J.P., Tarassi, K., Papadopoulos, I.A., et al. (2002) Shared Epitopes and Rheumatoid Arthritis: Disease Associations in Greece and Meta-Analysis of Mediterranean European Populations. Seminars in Arthritis and Rheumatism, 31, 361-370. http://dx.doi.org/10.1053/sarh.2002.31725

[3] Stahl, E.A., Raychaudhuri, S., Remmers, E.F., et al. (2010) Genome-Wide Association Study Meta-Analysis Identifies Seven New Rheumatoid Arthritis Risk Loci. Nature Genetics, 42, 508-514. http://dx.doi.org/10.1038/ng.582

[4] Song, G.G., Bae, S.C., Kim, J.H. and Lee, Y.H. (2014) Associations between TRAF1-C5 Gene Polymorphisms and Rheumatoid Arthritis: A Meta-Analysis. Immunological Investigations, 43, 97-112. http://dx.doi.org/10.3109/08820139.2013.837917

[5] Chorev, M. and Carmel, L. (2012) The Function of Introns. Frontier in Genetics, 3, 55. http://dx.doi.org/10.3389/fgene.2012.00055 